\title{
Knowledge-based Collaboration in Construction Industry
}

\author{
Dr Mikel Sorli ${ }^{1}$, Iñigo Mendikoa ${ }^{1}$, Juan Pérez $^{1}$, Dr Antonio Soares ${ }^{2}$, Ljubisa Urosevic ${ }^{3}$, Dragan \\ Stokic $^{3,}$ Jorge Moreira ${ }^{4}$, Helena Corvacho ${ }^{4}$ \\ ${ }^{\text {I} F u n d a c i o ́ n ~ L A B E I N, ~ C / ~ G e l d o ~-~ P a r q u e ~ T e c n o l o g i c o ~ d e ~ B i z k a i a, ~ E d i f i c i o ~ 700, ~} 48160$ - Derio (Bizkaia), \\ Spain, \{sorli,mendikoa, juan\}@labein.es \\ ${ }^{2}$ INESC Porto - Instituto de Engenharia de Sistemas e Computadores do Porto Campus da FEUP, Rua \\ Dr. Roberto Frias, $n^{o}$ 378, 4200-465 Porto, Portugal, als@fe.up.pt \\ ${ }^{3}$ Institut für angewandte Systemtechnik Bremen GmbH, Wiener Str. 1, D-28359 Bremen, Germany, \\ urosevic@atb-bremen.de \\ ${ }^{4}$ Instituto da Construção, Campus da FEUP, Rua Dr. Roberto Frias, Ed. H Sala 301, 4200-465 Porto, \\ Portugal, jmfcosta@fe.up.pt
}

\begin{abstract}
The present paper focuses on the topic of Communities \& Networks but it also covers some issues on Collaborative Enterprises, Collaborative Processes \& Workspaces, and Business to Business Networks. It is based mainly on the Collective project Know Construct (COLL-CT-2004-500276) starting in March 2005. The project aims to develop a common internet-based platform for SMEs from the construction sector to provide an effective combination of two general functionalities: an innovative decision making support system regarding the products characteristics, applications and other consultancy services for SMEs' customers applying the "web enabled dialogue", and a system for SMEs to support an advanced form of co-operation through the creation of Knowledge Communities of SMEs in Construction Industry. The system supports the integration, management and reuse of the area specific knowledge via a common knowledge base. The system is intended to be used within the Associations to collect and exchange the business area specific knowledge among the members (SMEs) in a form of essential expertise, reachable anywhere, at any time.
\end{abstract}

\section{Keywords}

Knowledge Communities, Ontology, Semantic Web, Construction Industry.

\section{Introduction}

The construction sector is characterised by a high level of fragmentation, with a large number of participants in each construction project, being the large majority SMEs. To increase flexibility and profitability the bigger construction companies have significantly reduced the scope of their activity and consequently the number of employees, focusing on the core tasks of the construction process and subcontracting most of the work to specialised and smaller companies. The sector is still characterised by a low level of education (in average), low productivity, low quality and expensive maintenance and disposal, and the SMEs in this sector are confronted with two key problems:

(a) Construction industry (particularly SMEs) urgently needs to radically improve communication with their customers in order to provide better product support and services. The customers of this industry, often individuals or SMEs, require costeffective forms of consultancy and services - an innovative form of "web based dialogues". Classical simple Internet solutions often do not satisfy the needs of the SMEs in this sector.

(b) The support to integrated teams creation through an integrated and well tailored IT approach can lead to crucial advancing in this business area. Application of the state-of- 
the-art ICT solutions is necessary to assure higher efficiency of the co-operation and integration processes. Necessity of knowledge and competence integration for a successful responding to customers needs (e.g. organisation of "turn key" contracts) imposes a need of establishing the knowledge communities of SMEs in construction industry.

A narrow technical specialisation must be replaced by significantly wider technical competence through integrated teams as knowledge communities, followed by on time, within budget works completion.

Taking into account these needs the consortium comprising Construction Industry Associations, SMEs from the same area and RTD organisations from four European countries decided to initiate the research project with a main goal defined as development of an innovative solution of Internet Platform for Knowledge-based Customer Needs Management (CNM) and for Knowledge Communities Support (KCS) for SMEs in Construction Industry. It was identified that the professional associations may represent an ideal environment to provide such a solution. The goal is therefore to contribute to enable the European SMEs in construction sector to increase the application level of the state-of-the-art, knowledge-based ICT solutions in their business relations to customer and mutual co-operation.

In order to successfully meet the challenges described, the KNOW-CONSTRUCT project [1] consortium defined its main objective as the development of a common platform for knowledge based systems, which provides a combination of two general functionalities:

(a) Customer Needs Management (CNM) System as an innovative decision making support system regarding the product characteristics, product applications and related consultancy services, and

(b) Knowledge Community Support (KCS) System as a System to support an advanced and efficient formation of communities of SMEs in construction industry, through their specific knowledge integration, management and reuse via a common knowledge base.

The platform will be owned by associations, which will offer to their members possibility to establish individual CNM systems and take benefit of Knowledge Community Support System.

The business objectives can be summarised as improved innovative technical support to product and service users (customers), wider technical competence required to satisfy customer needs (e.g. for "turn-key" solutions delivery), through closer co-operation and knowledge exchange among SMEs within knowledge communities, including improved quality/price ratio and on time completion of increasingly complex tasks.

\section{Relationship with the state-of-the-art}

In the recent past, many efforts were made to produce standard systems for construction products classification such as in the European project eConstruct where the bcXML, a XML version for Building and Construction Industry was developed and an appropriate taxonomy was introduced.

In order to establish an effective combination of e-KCS and e-CNM systems for SMEs several RTD topics are addressed: e-collaboration, ontologies, knowledge management including methods for knowledge acquisition, structuring, and retrieval, eCNM approaches, trust and security, tele-working technology, etc. The consortium carefully studied these topics in order to identify approaches that are most appropriate.

\subsection{Ontology development}

Ontology building has to serve as a basis for the knowledge representation from the related domains in Construction Industry. In more domain-specific such as construction industry there are currently several research experiences. The most important are the e-COGNOS [2] and eConstruct [3] projects. Ontologies attract high attention of RTD community but their 
application in practice is not still wide spread and additional RTD activities are needed to provide application oriented methods, whereby construction industry is not on the leading edge. Especially what is needed is a means for continuous update/maintenance of ontologies enabling long life of knowledge systems. KNOW-CONSTRUCT, plans to reuse or extend ontologies that can be applied in its context, ontology mapping approach is followed, which allows relating a portion of the source ontology to the target ontology's entities, transforming instances from the source ontology into instances in the target ontology.

The strategy to be followed therefore aims mainly at identifying, evaluating and reusing existing semantic resources, like the ontologies in the construction area, due to the advantages their reuse offers: more simple design, more reliable knowledge sharing and more clearly semantic representations. It was decided to deal with the very concrete reality of the SMEs of each country and with each Construction Industry Knowledge (CIK) Community of KCS system would need to have a local ontology that would answer the KC partners' particular professional and cultural needs and attend to its social context of use. It was decided to take this fact into account and look at this possibility as part of the standardised way to develop ontologies in the sector, but in such an away as to keep a common central ontological content (structure, attributes, relations, etc.) from where to derive the more specific ontologies. The development of this more specific ontology will, thus, be based on a larger, upper level ontology - the CIK Ontology, where all the central concepts of the construction area are structured. Therefore the solution proposed is to develop an inter-organizational knowledge management system for CIK Communities which will be built upon distributed ontologies locally managed and centrally integrated with CIK Ontology. The central ontology (CIK ontology) reflects standards and related classification schemes in the industry and the local ontologies will account for the individualised SME conceptual schemes, i.e. they will be strongly related to the consortium partners' needs.

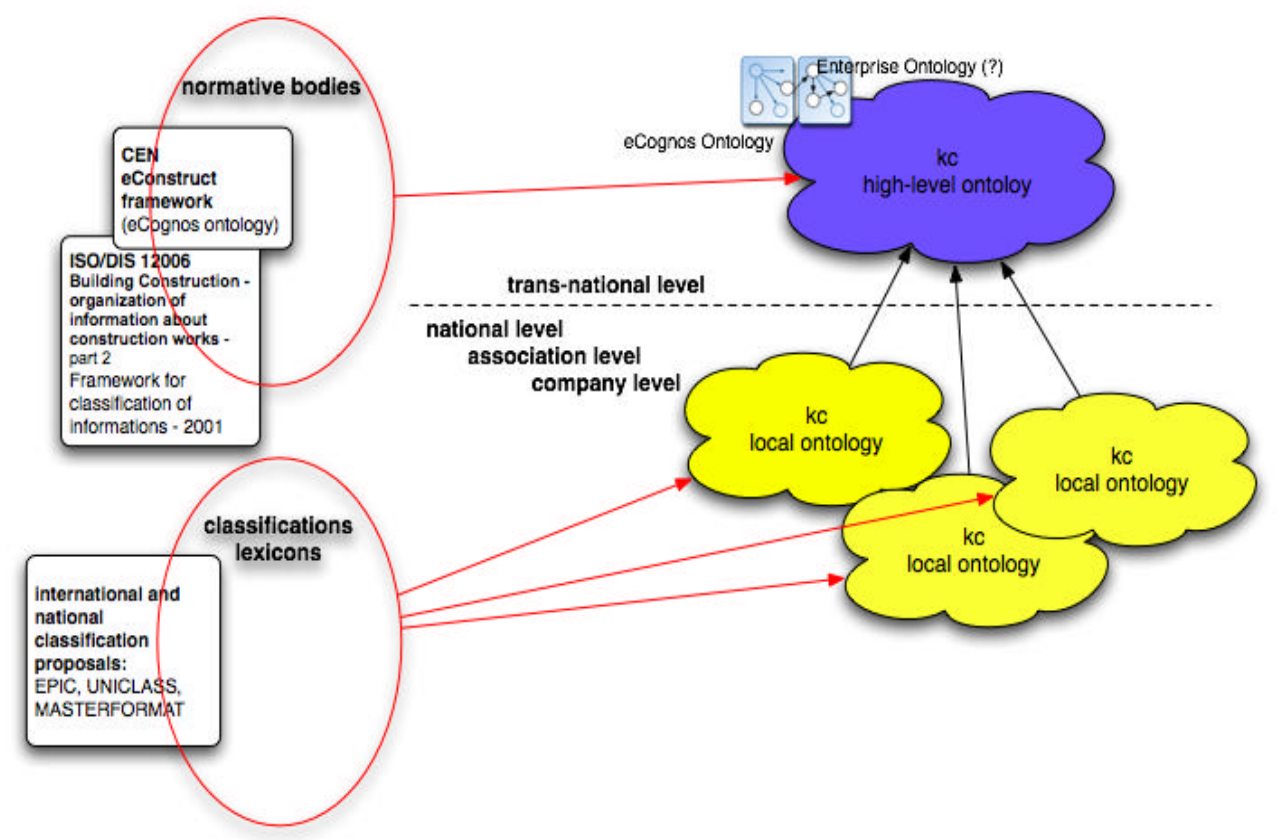

Figure 1 - General structure of the KC (Know-Construct) ontologies

\subsection{Software}

A survey of the SW solutions available in construction industry was carried out. The most relevant applications are the $\mathrm{B} 2 \mathrm{C}$ solutions that provide services to e-communities such as: customer relationship management, front office applications automation and business intelligence, as well as B2B solutions offering support for collaborative work. The relevance is in the concept of providing knowledge-based services to customers and information exchange among partners using Internet technologies. No software that effectively combines e-CNM and 
e-KCS functionality and assures effective knowledge sharing among SMEs in the construction industry has been found.

From the technologic perspective, the system developed in this project will be independent on the operating system. All the technology that will be used is either implemented in the Java platform or uses standard protocols for the exchange of information. This will allow for the portability between hardware, thus reducing the risk usually associated with the scalability of web based applications. The protocols used are either those defined by the W3C or any other "de facto" standard that is widely accepted and used. This includes amongst others: HTTP, SOAP and DIG. In addition to this, we also include in the realm of standard protocols, any link to databases (JDBC), query languages (SWRL, SPARQL, SQL) or even data transfer (XML).

\subsection{Expected Innovation}

Starting from the stated business and technical objectives and basing on the analysis of the stateof-the-art and current standards [4][5], the main problems addressed leading to innovations are:

- Methods for creation of Knowledge Communities of SMEs in construction industry, where the Associations get a crucial role.

- To establish or re-use an adequate domain related ontology [6], as well as classification system for this sector applicable in SMEs environment.

- An inter-organisational practicable knowledge management system for Construction Industry Knowledge Communities.

- New forms for a representation of the experience-based knowledge, very much present in construction industry.

- An open-architecture Internet based platform for a combination of the two basic functionalities.

- To provide a "web based dialogue" between SMEs and their customers aiming at an interactive decision support tool to be used for customer problem solving.

\section{Business scenarios}

The scenarios described below are related to some of the most probable situations in everyday work of the IAG member SMEs, where the KNOW-CONSTRUCT system is intended to provide support.

\section{Scenario 1: "Search for product information/documents"}

Potential individual customer or SME looks for an information about a construction product. The user logs-on in eCNM system and selects the product through the product categories/groups according to the ISO 12006-2/EPIC table. After navigating through an appropriate number of steps (in each step an appropriate number of selection boxes/lines are offered) a set of products is presented. The user chooses one of the entries of the result set to get more detailed information. The product's/manufacturer's web-site is displayed with a detailed information, providing also links to documents with more specific information about the product, application notes and other product related knowledge.

Scenario 2: "Search for construction work (service) information"

Potential individual customer or SME looks for information about a specific construction work (service) provider. The user logs on in eCNM system, and asks for "construction work (service)" and gets the list of services by type of work according to ISO 12006-2. Corresponding set of construction works/services is presented. The user chooses one of the entries of the result set to get more detailed information about the work/service forms and the data on the specific SME provider. The construction work (service) is displayed with detailed information including links to the service providers and documents with specific information about the construction work (e.g. necessary accompanying works, materials, supplier 
references etc).

\section{Scenario 3: "Search for Partner"}

SME user - IAG member, looks for the partner with specific competence (complementary or identical) in order to answer to the tender for the project overriding its power. The user logs on in eCNM system, and enters "Search for partner". The three categories of competence (1) Products (2) Works and (3) Entities/Objects are offered. Each one can be searched through as described above. The composite list of competences (e.g. x, y ,\& z from (1); t, b \& g from (2) and s from (3)) is processed by the system - partners searched. System also looks for the existing offers of other IAG members and sends the invitation/call to participate to the (automatically and/or manually) selected potential partners. Initial contacts are possible through the system.

\section{Scenario 4: "Customer Feedback"}

The system user - customer wants to insert some specific feedback related to a product implementation or functionality or to an improvement of characteristics. In order to facilitate the feedback insertion process and to subsequent analysis of the inserted comments/suggestions and their structuring, the customer will be lead through the above described CI areas up to the specific product, service or other topic. During the feedback insertion customer can use some of the above described functionalities as support for precise feedback definition.

\section{Scenario 5: "Navigate legal information"}

A works supervisor needs to have an idea of the legislation related with a given construction area. He/she does not know exactly what and where to look after the needed information. She then uses the semantic navigation facility of KCS that enables to browse set of legal documents and notes by according to several categories and perspectives: KC user selects "Navigation" from the system options; The system presents a top level graphical view of the classification scheme; The user selects "Legislation" from the top level categories; The system presents the next levels of the classification (Legislation subcategories); The user continues to go down the hierarchy until she finds a category that interest her; The user selects the option to show the content classified in the legal category previously selected; The system shows a list of content.

\section{Scenario 6: "Register problem/solution"}

A member of a company belonging to the association finds a solution to a given problem to be relevant for the community. He/she looks in $\mathrm{KC}$ if something similar is already available in the KCS system, possibly navigating through several problem categories. In the case of being a new solution, or a new problem, the user introduces the information about it being assisted by the system in finding the right category to classify it.

Scenario 7: "Consolidate product's application experiences “

A community member needs to gain some insight regarding possible problems in the application of a given construction product. For that, he/she needs to search for feedback from customers from all the companies belonging to the community. This way, she can find some recurring application patterns and correlate them with the problems reported. This is an example of how individual CNM collected information can be shared in the KCS community and be used by the community members.

\section{Overall system description}

The overall functional description of the system is represented in figure 2 above, showing some of the main general functionalities of both CNM and KCS modules. 


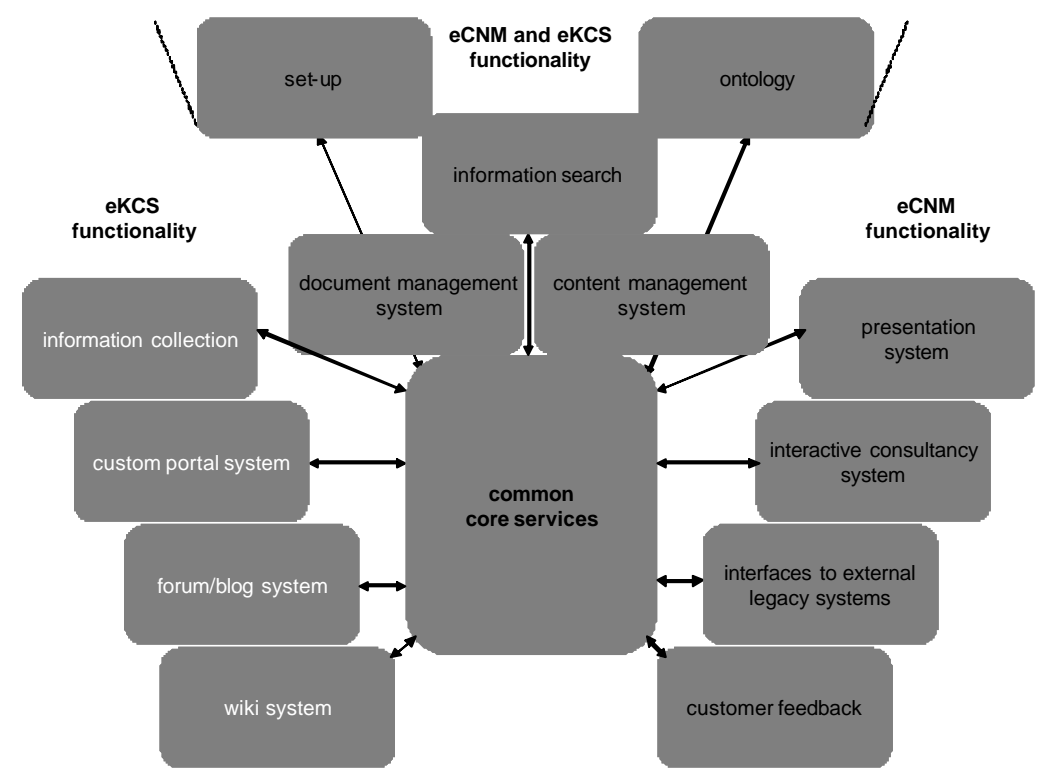

Figure 2: Overall Functional system description

The system architecture is described in the figure 3 below. The three main elements represent the KCS, CNM and inference back-end. The KCS and CNM will be connected via the CAPI which will allow client applications to request services and obtain results. The back-end will be responsible for the management and manipulation of the OWL schema and instance data. It will also provide basic administrative functions that will allow the management of the OWL.

Both the KCS and CNM elements represent complete applications. They provide not only the services and functions required but also the user interface. All OWL related functionalities required by these elements however will implemented by the $\mathrm{KC}$ back-end. Any additional functionality that is required will be specified and implemented for the KCS and CNM elements specifically including any services required for tagging and querying.

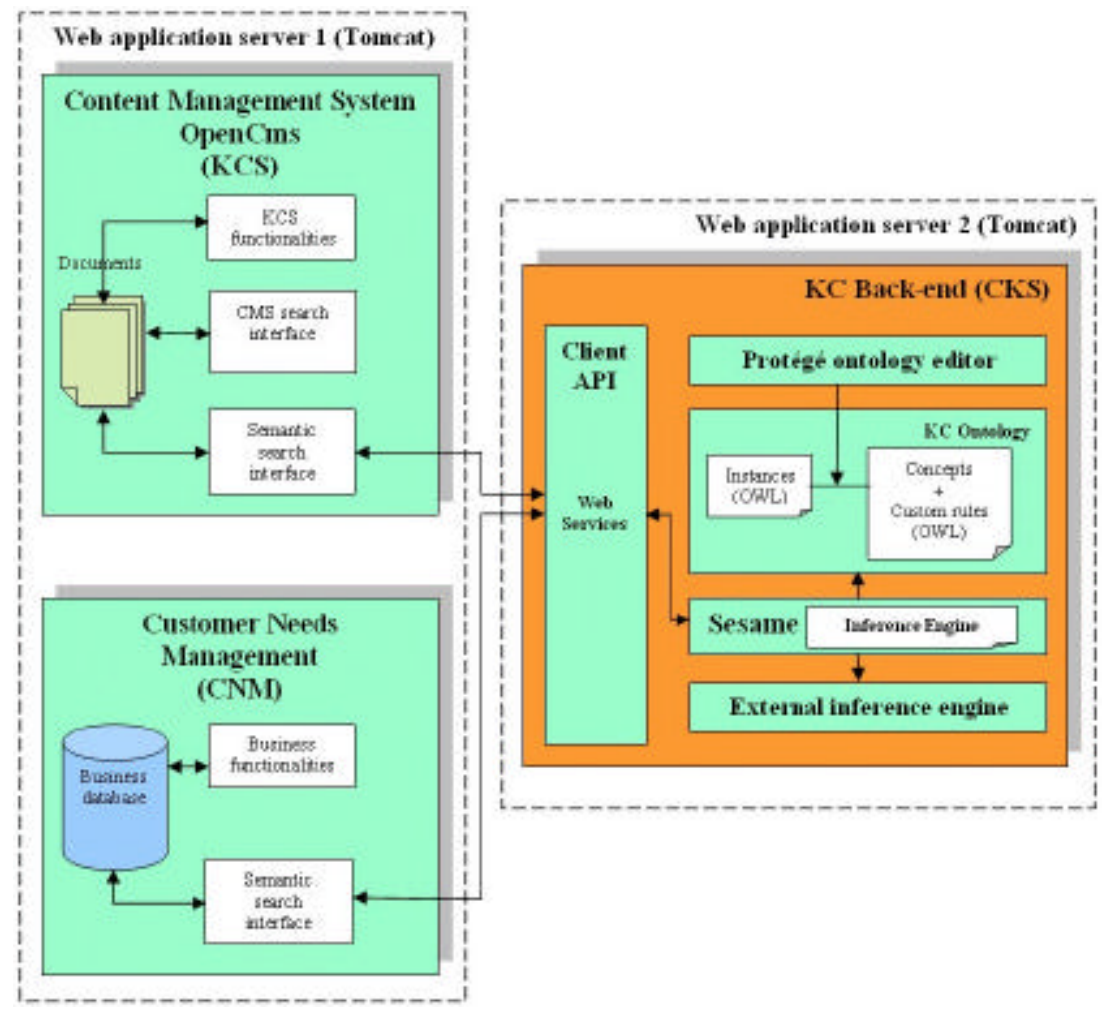

Figure 3: Overview of System Architecture 
The system is divided into three main components. The first is a set of software modules, interfaces and services that is used across the system. We shall refer to this component as the Common Knowledge Sub-System (CKS). This sub-system will include:

- A knowledge base schema (OWL Schema): this schema contains the description of the concepts supported by the KCIS. It will hold a set of classes, properties and relationships. In addition to this, a set of restrictions will also be used to better describe the concepts and their interrelations.

- A knowledge base instances (Information in the form of an OWL instance source): this data source will hold the instances of the classes described with the knowledge schema. Initially it will provide test data for the queries but will later also include the documents that have been labelled (or tagged) by the user community.

- Inference engines: one or more inference engines will be used to check the consistency of the ontology where is the ontology described and also allow for the querying of the instance data. This module may also include a set of inference rules that may (in conjunction with the OWL classes and restriction) allow for the querying of the data.

- Query engines: one or more query engines will be used to accept, parse and query the instance data. Such queries include simple traversal of the RDF or OWL graph. It will also include a set of queries that will allow a user to flexibly obtain information from (disparate and distributed) data sources.

- A client API (CAPI): will provide a consistent interface between the CKS and any clients application, which will be implemented for the KCIS. This includes the deployment of Web Services in order to communicate the CKS, more concretely the ontology, with the external applications such as the KCS and the CNM. The client API should be considered a communication request-response service where the external applications will ask for predefined and published services, which are processed by the client API, and will get a response under a defined format (XML). From the point of view of the external applications, they will only need to know what to get from the ontology in order to invoke the needed service. The client API will handle all the operations with the ontology and will return the needed data to the requesting application.

- Ontology Container (Sesame) API: is an API that will process requests from the CAPI (see point above). Essentially it receives requests to either manipulate or query the knowledge base (both schema and instance data) and accordingly return a result code or the data obtained from OWL data sources.

The system specification identified until now may be summed up into the following set of core functionality:

- Document storage and management: the basic information unit is a document. A document is any text, image file or any sound, video snippet that can be made available in electronic form. All this information will be made accessible through a web browser. Because many of these documents will be created, imported and changed within the KCIS system itself, it will therefore also be able to store and manipulate these documents. A Content Management System (CMS) will be used for this purpose.

- Document tagging or labelling: every document will describe a given number of concepts and be in some way or another related to other concepts. We say related in "some way or another" because a document may not directly describe or refer to a concept in order for it to be related (for example a product may have certain legal 
requirements that are not indicated in its marketing literature). Such documents and concepts will be manually linked to the concepts. A document (as a whole or one of its elements such as a section or paragraph) will be labelled or tagged with a given property or relation. Note that in addition to the KC specific constructs additional meta-data may be used which will provide greater query capabilities (For example we can use the Dublin Core meta-data to indicate the author of the document or we may create additional meta-data to record for example who tagged the document). The result of the tagging is an OWL instance store. Documents themselves will be resources with a given URI (Uniform Resource Identifier).

- Data query: once the documents have been tagged (or labelled), they can be searched. Simple or parametrized queries are received and processed by the KCIS's back-end. The data is collected and returned in a document according to a pre-defined format. Besides the operational issues regarding query processing, this document will also contain examples of queries and their parametrization. Operational issues include data transfer formats, selected query languages, selected query engines, and any decisions that influence efficiency and correctness.

- Data Visualization: information will first and foremost be made available visually whether it is text or images. Visualization may also potentially includes the playback of video and sound. The KCIS must provide a means to open and view documents whether for labelling or standard information consumption, view the ontology (including metadata) in order to label or tag the parts of the documents and finally provide a means to list and analyse the query results.

\section{Conclusions and future work}

The development of this customer needs and community knowledge management platform, including the application procedures of construction products and best practices using the identified norms and classification system, is considered by the KNOW-CONSTRUCT consortium partners (which includes industrial SMEs and Associations of Construction Industry in four European countries), as an important contribution for the improvement of the performance of the SMEs in the sector.

After the project has been running 12 months, the system design has been completed and the system prototype is now under development. In order to perform the assessment and validation of the KNOW-CONSTRUCT solution metrics will be defined to enable a quantitative assessment of the project progress and the results achieved against the industrial requirements. Measurements will be carried out from both the business and the technical point of view.

\section{Acknowledgement}

Project "Internet Platform for Knowledge-based Customer Needs Management and Collaboration among SMEs in Construction Industry" is funded by the European Commission under the Collective Research program (Project contract: COLL-CT-2004-500276). The authors wish to acknowledge the contribution of the consortium partners.

\section{References}

[1] KNOW-CONSTRUCT project (COLL-CT-2004-500276), available at http://www.know-construct.com/

[2] e-COGNOS Public Deliverables, available at http://www.e-cognos.org/

[3] eConstruct Public Deliverables, available at http://www.bcxml.org/

[4] CWA3 - CEN Workshop Agreement, European eConstruction Ontology (EeO), 2004. Documents produced by the CEN/ISSS eConstruction Workshop, Brussels, 2004.

[5] ISO 12006-2 Building construction - Organization of information about construction works - Framework for classification of information, DIS version 2001.

[6] W3C, 2002. Requirements for a Web Ontology Language, available at http://w3.org/ 